\title{
The Unusual Southern Hemisphere Stratosphere Winter of 2002
}

\section{PAUl A. NEWMAN}

NASA Goddard Space Flight Center, Greenbelt, Maryland

\section{ERIC R. NASH}

Science Systems and Applications, Inc., Landover, Maryland

Submitted 1 April 2003.

Corresponding author address: Paul A. Newman, Mail Code 916, NASA Goddard Space Flight Center, Greenbelt, MD 20771.

E-mail: newman@code916.gsfc.nasa.gov 


\begin{abstract}
The southern hemisphere stratospheric winter of 2002 was the most unusual winter yet observed in the southern hemisphere climate record. Temperatures near the edge of the Antarctic polar vortex were considerably warmer than normal over the entire course of the winter. The polar night jet was considerably weaker than normal, and was displaced more poleward than has been observed in previous winters. These record high temperatures and weak jet resulted from a series of wave events that took place over the course of the winter. The first large event occurred on 15 May, and the final warming occurred on 25 October. The propagation of these wave events from the troposphere is diagnosed from time series of Eliassen-Palm flux vectors. The wave events tended to occur irregularly over the course of the winter, and pre-conditioned the polar night jet for the extremely large wave event of 22 September. This large wave event resulted in the first ever observed major stratospheric warming in the southern hemisphere. This wave event split the Antarctic ozone hole. The combined effect of the wave events of the 2002 winter resulted in the smallest ozone hole observed since 1988.
\end{abstract}




\section{Introduction}

During the fall of 2002, the Antarctic ozone hole was unusually disturbed. First, the ozone hole was considerably smaller than has been observed during the early September period. Second, the ozone hole split into two parts on 22 September 2002. We will show that the reason for this unusual behavior was not a result of chlorine chemistry, but because of the stratospheric temperature and dynamics that occurred over the winter period. There are two necessary conditions for causing the Antarctic ozone hole: high levels of halogens (specifically chlorine and bromine, see WMO 1995), and temperatures cold enough to form polar stratospheric clouds (PSCs).

The impact of temperature on Antarctic ozone losses has always been considered to be of secondary importance because temperatures are always cold enough to form extensive PSCs, and the southern hemisphere (SH) has small interannual variability during winter (Randel 1992). It has long been recognized that Antarctic September temperatures exhibit extremely cold conditions (i.e., temperatures < $193 \mathrm{~K}$, the approximate formation temperature for PSCs). For example, Court (1942) noted the very cold winter conditions from balloon observations during September 1940 at Little America $\left(78^{\circ} \mathrm{S}\right)$ and remarked “... since in general, soundings ended prematurely as soon as they reached $-80^{\circ} \mathrm{C}$ level, apparently the limit of balloon elasticity." In contrast, Arctic temperatures are both warmer and more variable. This variability will occasionally results in large Arctic ozone losse (e.g., 1997), or virtually no ozone loss at all (e.g., 1999)(Rex et al., 2002).

In this paper, we will describe the evolution of the 2002 SH stratospheric winter. First, the winter of 2002 evolved from a larger than normal series of wave events that spanned the entire winter. These waves varied in strength and duration, the largest and most spectacular of these events was the major warming of 22 September 2002 that split the ozone hole into two pieces (Thompson et. al. 2003). Second, each wave event acted to warm the region near the polar night jet, i.e., the winter was characterized by a series of minor and a single major warming from waves. The total wave energy propagating into the stratosphere quantitatively explains 
the much warmer than normal temperatures during September. Third, the polar vortex broke down earlier than normal. The typical warming of the SH has a regular downward progression that usually begins in July in the upper stratosphere and ends in late November in the lower stratosphere. In 2002, this same downward progression of warming also occurred, but at an accelerated rate because of the greater number of wave events over the course of the winter. Fourth, eddy variance in the troposphere was large, but did not exhibit a systematic pattern over the winter period. However, the wave 1 pattern was clearly anomalous during the winte of 2002 from the tropics to the high latitudes.

\section{Data}

The NCEP/NCAR reanalysis (NNR) data are used as the basis for this work. We restrict these analyses to data after 1978 because of the poorer quality of the NNR data in the stratosphere prior to the inclusion of satellite observations in 1979 (Mo et al. 1995; Kanamitsu 1997; Santer et al. 1999; Marshall 2002). In addition, certain quantities that we calculate, such as the heat flux, exhibit considerable uncertainty in both hemispheres (Newman and Nash 2000; Randel et al. 2002). Northern hemisphere (NH) flux values from a variety of analyses show differences of $\pm 15 \%$ (Newman and Nash 2000), while a comparison of the SH heat flux between the NNR and ECMWF analyses by Randel et al. (2002) show large differences. Nevertheless, the differences between the year 2002 and past years are so large that 2002 analysis differences with climatology are small compared to the overall analysis errors. Unless otherwise noted, for display purposes the time series shown herein have been smoothed to reduce day-to-day variability.

\section{Results}

Temperatures near the jet axis were higher than normal in the polar stratosphere during the entire winter. The black line in Figure $1 \mathrm{C}$ displays the zonal mean daily temperature at 
$65^{\circ} \mathrm{S}$ and $50 \mathrm{hPa}$ during the 2002 winter. Up to mid-May, temperatures at $65^{\circ} \mathrm{S}$ (near the core of the vortex) were near normal. There is a slight increase in mid-May, followed by a relaxation back to normal temperatures until mid-June. By late June, temperatures were well above normal, and were regularly higher than any values observed at a comparable time over the last 24 years. In late-September, the temperature dramatically increased to values that were $15 \mathrm{~K}$ warmer than average.

In contrast to the temperatures near the jet axis, temperatures in the core of the vortex (near the South Pole) were only slightly higher than normal over the course of most of the winter. Figure $1 \mathrm{~B}$ displays the minimum daily temperature observed between $50^{\circ}-90^{\circ} \mathrm{S}$ at 50 $\mathrm{hPa}$ during the 2002 winter. Only in the late-September period does the temperature jump to a value that is higher than climatology.

Temperatures at higher altitudes inside the vortex were slightly colder than average during the winter period. Figure 1A displays the minimum daily temperature observed between $50^{\circ}-90^{\circ} \mathrm{S}$ at $10 \mathrm{hPa}$ during the 2002 winter. At these higher altitudes, the 2002 temperatures were generally below the climatological daily average (white line). The exceptions occur in early September and the late-September through October period. These colder than normal temperatures at higher altitudes suggest that PSCs may have been forming at altitudes much higher than normal during the 2002 winter.

The vertical structure of these warmer than normal temperatures near the edge of the vortex are displayed in Figure 2A. This figure is a height-time plot of the of the $2002 \mathrm{SH}$ zonal-mean temperatures near the core of the polar night jet $\left(55^{\circ}-75^{\circ} S\right)$. During the April to early-May period, temperatures are near normal. A minor warming occurs in mid-May. By early June, temperatures begin to steadily increase with respect to the climatological average. By early July, these temperatures have increased to extremely high values, with further increases culminating in a major stratospheric warming on 22 September 2002. A major warming is defined by a reversal of the zonal-mean temperature gradient between $50^{\circ}-80^{\circ} \mathrm{S}$, combined with a reversal of the very strong $60^{\circ} \mathrm{S}$ zonal wind at $10 \mathrm{hPa}$ to easterlies values. 
The temperature differences from climatology became increasingly warmer over the course of the winter as a result of stepwise warmings, with some cooling after each warming. The warming appears to descend over the course of the winter. For example, the May and June warmings peak near or above $10 \mathrm{hPa}$, and only extend down to about $100 \mathrm{hPa}$, the August temperature anomalies peak below $10 \mathrm{hPa}$ and extend below $100 \mathrm{hPa}$, and the September major warming peaks near $30 \mathrm{hPa}$ and extends below $300 \mathrm{hPa}$.

The warming of the polar vortex collar region is directly controlled by the planetary waves that propagate upward from the troposphere into the stratosphere (Hartmann et al. 1984; Shiotani and Gille 1987). Figure 2B displays a 2002 time series of mid-latitude eddy heat flux at $100 \mathrm{hPa}$ for waves 1-3. As discussed in Andrews et al. (1987), the heat flux represents the vertical flux of wave energy. Over the course of the May-October period, we identify 11 significant wave events (Table 1). Vertical lines are superimposed between panels of Figure 2 to show the connection between the wave events and the warmings. The wave events are irregularly spaced with about a 1-3 week periodicity.

The waves not only impact temperature, but also the zonal-mean wind. Figure $2 \mathrm{C}$ displays the height-time plot of the zonal-mean zonal wind difference between 2002 and a 1979-2001 average in the $20^{\circ}-90^{\circ} \mathrm{S}$ zone. This figure effectively represents the deviation of the average jet strength from climatology. Each wave noted in Figure 2B impacts the strength of the jet in Figure 2C, with the 22 September wave completely reversing the westerlies to weak easterlies. Combined with the temperature increase in Figure 2A and Figure 1, it is clear that this event was the first major stratospheric warming yet observed in the southern hemisphere. However, it is important to recognize that the flow was first disturbed by the 15 May event, and was highly disturbed by mid-August.

The wave events over the course of the winter cumulatively acted to warm the polar lower stratosphere. Furthermore, this warming is fairly consistent with previous winters. Figure 3A displays the late-September polar temperature at $50 \mathrm{hPa}$ plotted against the average mid-latitude heat flux for a 53-day period prior to the temperature period. The two 
quantities are highly correlated. In essence, the cumulative effect of the waves over the August-September period acts to warm the polar region (see Newman et al. 2001 for a discussion of comparable effects in the Arctic). During 2002, the late-September temperatures were significantly warmer than any previous winter because of the unusual strength and duration of the wave events. Most of the impact of these wave events tends to occur near the edge of the vortex (near the jet axis). While the wave events are apparent in the core of the vortex (see Figure 1A), the effect is smaller than at the edge.

The cumulative effect of the waves also impacted the temperatures prior to the major warming shown in Figure 2A. Figure 3B displays the early-September polar temperature at $50 \mathrm{hPa}$ plotted against the average mid-latitude heat flux for a 53-day period prior to this temperature period. Again, note the excellent correlation. Early-September 2002 had the highest temperature with the highest mid-winter heat flux. While the 1986 heat flux was comparable to 2002, the flux in early winter was smaller than in 2002 . The major difference between 1986 and 2002 was the late-September 2002 major warming. The average heat flux in 2002 changes by about $5 \mathrm{~km} / \mathrm{s}$ by shifting the time average by 15 days, an indicator of the magnitude of the late-September 2002 wave event.

The spatial pattern of warm temperatures covers a considerable region of the $\mathrm{SH}$ by late August. However, as mentioned previously, the higher than normal temperatures were predominantly in the region near the jet core. Figure 4A shows temperature differences between July 2002 and a 1979-2001 climatology. The climatology is superimposed on the figure as white lines. Temperatures are much warmer near the climatological jet core and vortex edge. In the vortex core near the pole, temperatures are only 1-2 $\mathrm{K}$ warmer than climatology in the lower stratosphere, in agreement with the minimum temperatures shown in Figure 1B. In addition, the temperatures at higher altitude (above $20 \mathrm{hPa}$ ) inside the vortex were colder than normal, in agreement with the minimum temperatures shown in Figure 1A.

The zonal-mean wind was also very disturbed during July 2002, prior to the 22 September major warming. Figure 4B displays zonal-mean wind differences between July 2002 and 
climatology. The jet core was closer to the pole during 2002, and so the polar vortex was somewhat smaller. The zero-wind line in the lower to middle stratosphere was approximately $6^{\circ}-10^{\circ}$ closer to the pole in 2002 than the climatological average. The quasi-biennial oscillation (QBO) was in the westerly phase in 2002, as is apparent from the positive anomaly near the equator at 20-30 $\mathrm{hPa}$. In addition, winds in the upper stratospheric tropics were in the easterly phase (not shown on Figure 4B). The series of wave events shown in Figure 2B decelerated the jet as shown in Figure $2 \mathrm{C}$, leading to the smaller and weaker vortex by July and into September.

The Eliassen-Palm (EP) flux is an extremely useful diagnostic for evaluating the propagation of waves in the stratosphere. The EP flux vector points in the direction of the waves group velocity (Andrews et al., 1987). In Figure 5 the EP flux is shown in a vertical cross section (Figure 5A), and horizontal cross sections for $300 \mathrm{hPa}$ (Figure 5B) and $10 \mathrm{hPa}$ (Figure 5C). These EP flux vectors are only calculated from waves 1-3 (i.e., planetary scales). The vertical lines indicate the major wave events diagnosed from the $100 \mathrm{hPa}$ heat flux, as shown in Figure 2B. The top panel shows that over the course of the winter, these waves are propagating into the stratosphere from the lower-to-mid troposphere region. Generally the waves move upward and equatorward, where equatorward propagation is indicated by the vectors that lean towards the right on the panel. At $10 \mathrm{hPa}$, these waves are strongly propagating towards the equator. Prior to the mid-May period, these planetary scale waves do not appear to propagate into the stratosphere. Further, after the 23 September event, the waves also do not penetrate into the middle stratosphere, except for weak event in late-October. This late-October event produces the final warming that ended the winter.

In the $150-200 \mathrm{hPa}$ region of the stratosphere, the waves generally propagate upward and equatorward (see Figure 5A). The large exception to this case occurs in September during the major warming. A close inspection of Figure 5A shows that the waves are strongly propagating towards the pole over a broad altitude region extending into the mid-stratosphere. This convergence into the polar cap produces the sudden warming by rapidly decelerating the 
mean wind and warming the polar region. Further examination of the figure shows that the poleward propagation of this 23 September wave extends well into the troposphere.

As noted above, the wave event of 23 September showed propagation into the polar region over a very large vertical depth. Figure 5B displays the EP flux vectors on the $300 \mathrm{hPa}$ surface. On this figure, vectors pointing towards the left are upward propagation, while vectors pointing up are propagating equatorward. This figure shows that the upward propagation of waves in the upper troposphere occurs over a broad latitudinal extent $\left(35^{\circ}-65^{\circ} \mathrm{S}\right)$. Furthermore, nearly all of the wave events at $300 \mathrm{hPa}$ show some poleward wave propagation.

The wave events shown in Figure 2B did not exhibit a particularly coherent pattern over the course of the winter. For example, the wave flux events shown in Figure 2B are computed using only waves $1-3$. Table 1 shows the dominant wave numbers associated with each warming. The wave events of 15 May, 7 July, 18 August, and 24 October were all dominated by strong wave 1 patterns, while the 9 June, 31 July, and 5 September events were dominated by wave 2 patterns. While the eddy heat flux is dominated by wave 1, waves 2 and 3 cannot be neglected.

The eddy variability for the winter of 2002 is illustrated in Figure 6. Figure 6A displays the RMS wave amplitude as a function of altitude averaged in the $45^{\circ}-75^{\circ} \mathrm{S}$ region for waves 1-3. The RMS amplitude has been normalized by the square root of the density to emphasize the tropospheric wave activity. Again, the vertical lines indicate the peak heat flux periods at $100 \mathrm{hPa}$ show in Figure 2B. The figure shows that the heat flux peaks are typically accompanied by coherent waves from the mid-troposphere to $10 \mathrm{hPa}$, as was also shown in Figure 5A. Peak amplitudes at $300 \mathrm{hPa}$ typically precede the peak amplitudes at $10 \mathrm{hPa}$ by a few days. In the stratosphere, the wave amplitudes steadily strengthen over the course of the winter, reaching a maximum during the major warming in late-September. The wave events also do appear to conserve their amplitudes as the propagate upward. The waves generally have maxima in the troposphere, with a minimum in wave amplitude above the tropopause, and a second maximum in the middle-to-upper stratosphere. 
The planetary-scale eddy variability in the troposphere is concentrated in the $45^{\circ}-75^{\circ} \mathrm{S}$ region. Figure $6 \mathrm{~B}$ shows the RMS wave amplitude for waves $1-3$ at $300 \mathrm{hPa}$ in the geopotential height field. The wave-event maxima vary in both strength and latitude location over the course of the winter. A Hovmoller diagram of the wave 1-3 eddy field is shown in Figure 6C for a latitude of $60^{\circ} \mathrm{S}$ (gray horizontal line in Figure 6B). Note that the Hovmoller diagram is dominated by a wave 1 pattern, with the wave 1 peak centered at about $130^{\circ} \mathrm{W}\left(260^{\circ} \mathrm{E}\right)$, just west of the Antarctic peninsula. However, it is clear that the wave events primarily occur as episodic bursts of wave energy moving into the stratosphere on a 1-2 week time scale.

Height and stream function field deviations from a 1979-2001 climatology in the upper troposphere for the June-August period (not shown) suggest a possible wave train that is forced in the maritime continent region of the Western Pacific. Correlations of $100 \mathrm{hPa}$ heat flux averages with the mid-winter flow field suggests a relationship: viz., strong anti-cyclonic flow to the west of the Antarctic peninsula (approximately $60^{\circ} \mathrm{S}, 130^{\circ} \mathrm{W}$ ) is associated with large eddy activity propagation into the stratosphere.

We have investigated the propagation of the planetary-scale waves using a crosscorrelation analysis of the stream function. A cross-correlation analysis of the geopotential height field was used by Randel (1987) to investigate the propagation of planetary waves in the winters of 1983 and 1984. Figure 7 displays the coherence for wave 1 at $60^{\circ} \mathrm{S}$ and 100 $\mathrm{hPa}$ with wave 1 at various other altitudes and time lags. The coherence is effectively the correlation of wave 1 with other altitudes and times. We calculate this coherence using the period 1 May to 15 September 2002. This period is chosen to understand the wave 1 events prior to the late-September major warming. The strong coherence between the troposphere and $100 \mathrm{hPa}$ is evident, but showing a shift backward of $2-3$ days down to the surface. In addition, the peak of the coherence shifts forward in time at $10 \mathrm{hPa}$ by about 2 days. This tilt of the coherence is directly related to the upward propagation of wave 1 , with a wave propagation of approximately $6 \mathrm{~km}^{-1} \mathrm{day}^{-1}$. Plots for waves 2 and 3 show similar coherence, but with faster vertical propagation. This coherence pattern is in good agreement with the EP 
flux propagation shown in Figure 5, and confirms the time lag in the wave events between the middle troposphere and middle stratosphere.

We can further trace the behavior of waves in the middle-lower troposphere using wave 1 coherence plots with various time lags. Figure 8 displays the coherence between the $150 \mathrm{hPa}$ and $30^{\circ} \mathrm{S}$ point and all of the points on a latitude vs. pressure-altitude plot for time lags of $-4,0,+2$, and +4 days. This figure shows the coherence of the tropical wave 1 pattern with the mid-latitude lower tropospheric wave 1 . The pattern indicates strong coherence across the upper tropospheric tropics from $20^{\circ} \mathrm{N}$ to $30^{\circ} \mathrm{S}$ (Figure 8A) at a -4-day lag. This suggests cross-equatorial wave propagation (as indicated by the arrow). The 0 -day and +2 -day lag panels (Figure 8B and C) show a correlation of the upper-tropospheric sub-tropics $\left(30^{\circ} \mathrm{S}\right)$ has developed with wave 1 at $700 \mathrm{hPa}$ and $55^{\circ} \mathrm{S}$ (indicated by the arrows). The +4-day lag panel (Figure 8D) shows a weakening wave 1 in the tropics, with intensifying coherence from 700 $\mathrm{hPa}$ into the stratosphere at $60^{\circ} \mathrm{S}$. This figure shows that the stratospheric wave 1 pattern is strongly influenced by a tropical wave 1 on a time scale of about 8 days.

Because of the strong coherence between wave 1 at $150 \mathrm{hPa}$ and wave 1 at $700 \mathrm{hPa} 2-3$ days earlier, we have examined the long term behavior of the stream function at $700 \mathrm{hPa}$. Figure 9A displays the average wave 1 amplitude between $50^{\circ}-70^{\circ} \mathrm{S}$ at $700 \mathrm{hPa}$ for each year from 1979-2002 for a 1 May to 15 September average. In this figure, 2002 stands out as the record value. In addition, 1988 and 1992 also stand out as maxima, and these were also years with active ozone holes. As noted above, the wave 1 amplitude at $700 \mathrm{hPa}$ is related to the tropical wave 1 amplitude with about a 4-day lag. Figure 9B displays the average wave 1 amplitude between $10^{\circ}-20^{\circ} \mathrm{S}$ at $300 \mathrm{hPa}$ for each year from $1979-2002$ for the same 1 May to 15 September average (no time lag). Again, the wave 1 amplitude in the southern tropics is a maximum. The two time series have a reasonable 0.51 correlation, which for 24 years that are independent is significantly different from zero at the $99 \%$ confidence level for a 2 -sided Student's T-test. However, this correlation only explains about $26 \%$ of the variance in the wave $1700 \mathrm{hPa}$ variance. 
We have also examined the coherence of waves 2 and 3 during the winter of 2002 . Both waves 2 and 3 shows distinct vertical propagation into the stratosphere, but only a weak relationship to the subtropics.

\section{Discussion}

The southern hemisphere stratosphere was extremely disturbed during the winter of 2002. The culmination of this disturbed stratosphere was the major warming of 22 September 2002. There are two hypotheses to explain this increase in stratospheric wave activity: (1) excessive tropospheric wave forcing in 2002 propagating upward into the stratosphere, and (2) an anomalous mean flow that allowed moderate tropospheric waves to more easily propagate into the stratosphere.

The tropospheric wave forcing was higher than average during the 2002 winter. For the April-September period, the $200 \mathrm{hPa}$ heat flux for waves $1-3$ in the $40^{\circ}-70^{\circ} \mathrm{S}$ region was $50 \%$ stronger than climatology, and substantially stronger than any previous year. If we exclude September from this average, then the heat flux was still about $40 \%$ stronger than climatology, but comparable to both 1992 and 1995 . While it was higher than average, wave forcing was not at record levels near the tropopause. However, as was shown in Figure 9A, wave 1 was anomalously larger in the lower troposphere. This higher than normal wave 1 is related to higher than normal wave 1 in the tropics. Both waves- 2 and 3 were also higher than normal in 2002 , but did not show any relationship to tropical wave forcing.

As noted above, wave 1 in the lower troposphere was stronger than normal during the winter of 2002. From the coherence in Figure 8, wave 1 at this sub-tropical point is strongly related to both northern subtropical waves. This suggests that wave energy propagates southward across the equator and downward into the southern mid-latitude troposphere, reinforcing the wave 1 in the lower troposphere, which then propagates upward into the stratosphere.

The weaker stratospheric winds and warmer temperatures seen in 2002 are clearly 
consistent with greater wave forcing (e.g., the modeling study of Taguchi and Yoden 2002). However, the generation of these large-scale stratospheric waves is difficult to explain. Large wave forcing from the SH troposphere is consistent with the modeled dynamic forcing discussed in Scinocca and Haynes (1998). In their model, stratospheric wave activity was forced by non-linear wave-wave interaction of tropospheric baroclinic waves. This wave-wave interaction was characterized by waves with relatively slow phase velocities embedded in wave packets with large group velocities. However, they noted that it was not always possible to associate forcing of waves with bursts of upward EP flux. An analysis of the upper tropospheric eddies does not reveal clear evidence of excessively strong wave packets.

The wave propagation is controlled by the index of refraction, which is proportional to the meridional potential vorticity gradient and inversely proportional to the zonal-mean wind minus the wave's phase speed (Dickinson 1968). As was noted in Figure 2B, the zero-wind line was $6^{\circ}-10^{\circ}$ closer to the pole in 2002 . At $10 \mathrm{hPa}$, the $20 \mathrm{~m} \mathrm{~s}^{-1}$ zonal-mean wind is usually found at $36.4^{\circ} \mathrm{S}$. In 2002 , the $20 \mathrm{~m} \mathrm{~s}^{-1}$ zonal-mean wind was found at $43.6^{\circ} \mathrm{S}$. During August 2002 (preceding the major warming), the polar-night jet was the weakest yet observed, and the equatorial flank of the jet was located at the most southerly latitude since 1988 . This implies that slow moving planetary waves would encounter a critical line at a more southerly latitude than is normally found in past years. This anomalous jet structure was also found in earlier months of 2002. For example, in April (prior to any wave events noted herein), the subtropical zonal winds in the upper stratosphere were anomalously easterly, and the jet was a few degrees closer to the pole than normal. The structure of the zonal-mean wind during 2002 was anomalous, suggesting that wave propagation was highly anomalous during 2002.

The impact of tropical and extratropical winds on stratospheric variability has been shown by Holton and Tan (1982). They showed that the QBO impacted wave propagation in the NH stratosphere, and hence the interannual variability of the polar vortex. Gray et al. (2001a) extended this work to show that upper stratospheric winds dramatically impacted NH polar vortex variability, while Gray et al. (2001b) utilized a model to show 
that mid-to-upper stratospheric equatorial winds were necessary to explain the Holton-Tan relationship. Scott and Haynes (1998) have also used a model to demonstrate that disturbed winters are accompanied by late-fall, early-winter easterlies in the subtropics. Indeed, such easterlies were observed in 2002 in the tropics in association with a westerly QBO at $30 \mathrm{hPa}$. In fact, temperatures near the vortex edge in early September are highly anti-correlated with tropical winds in the upper stratosphere.

In addition to the stratospheric index of refraction, the index of refraction at the tropopause was slightly more conducive to waves propagating into the stratosphere. Chen and Robinson (1992) suggested that the refractive index at the tropopause acted as a valve for the propagation of planetary waves. Figure 10 is a plot of the $45^{\circ}-75^{\circ} \mathrm{S} 100 \mathrm{hPa}$ heat flux plotted against the quasi-geostrophic potential vorticity meridional gradient as a function of year averaged between 400 and $100 \mathrm{hPa}$, and $40^{\circ}-60^{\circ} \mathrm{S}$. The quantities are time averaged from 1 May to 15 September for each year. The correlation between the heat flux and PV gradient is 0.77 . If we perform a multiple regression of the $500 \mathrm{hPa}$ heat flux (tropospheric wave forcing) and the PV gradient (index of refraction) against the $100 \mathrm{hPa}$ heat flux, the correlation increases to 0.9 . This confirms the basic hypothesis that the wave energy that has entered the stratosphere is directly related to the tropospheric forcing and the valving near the tropopause controlled by the index of refraction.

\section{Summary and Conclusions}

The 2002 southern hemisphere winter had perhaps the most interesting stratospheric conditions yet observed since stratospheric observations first began in the 1940s. This winter had the only major stratospheric warming ever observed in the SH. While such major warmings are relatively common in the $\mathrm{NH}$, the typical warming of the SH only occurs in the final warming during the October-November period. The warmer temperatures and stronger wave dynamics significantly impacted the Antarctic ozone hole.

The 2002 hole was the least severe since 1988 . The 2002 wave events dramatically 
impacted the Antarctic ozone hole (Stolarski et al. 2003). Using TOMS total ozone, the size of the hole was already small during the period preceding the major warming. This smaller size is consistent with the warmer temperatures at the vortex edge. The major warming then split the Antarctic ozone. The ozone hole had an early disappearance in response to the final wave event on 24 October.

The mean temperature of the polar lower stratosphere was much warmer than normal during the winter of 2002 . The temperature of the Antarctic stratosphere was directly related to the wave forcing events observed over the course of the winter. While strong eddy forcing of the SH stratosphere is not common, the resulting high temperatures were generally consistent with this strong forcing in both magnitude and timing. Hence, the higher than normal polar temperatures did not result from any direct radiative forcing mechanism.

The SH stratospheric wind fields were also highly anomalous during the winter of 2002. This was especially true following the major warming of 22 September. However, it is also clear that the winds were abnormal as far back as April. The anomalous easterlies in the mid-to-upper stratosphere intensified over the course of the winter, with the jet becoming progressively weaker and moving closer to the pole. The wave events up through early September preconditioned the wind fields to allow the major warming of 22 September.

The winter dynamics of 2002 is directly responsible for the unusual ozone hole, and the unusual dynamics appears to result from higher than average wave forcing from the troposphere. In particular, we find that the larger than normal propagation of planetary scale waves into the stratosphere was caused by 2 main factors: (1) stronger than normal levels of planetary wave 1 in the southern mid-latitude lower troposphere, and (2) a lower than normal index of refraction at the tropopause that allowed more wave penetration into the stratosphere. The stronger than normal wave 1 appears to result from strong forcing of wave 1 in the tropics of both the northern and southern hemispheres.

Certain question remain unresolved. First, what is the mechanism for the forcing of the tropical wave 1? Correlation with sea surface temperatures and the southern 
oscillation index do not show significant correlations with the tropical wave 1 in the upper troposphere. Australia experienced a severe drought and the monsoon rainfall was $19 \%$ below normal during 2002 (NOAA/NCDC Climate of 2002 Annual Review, http://hwf.ncdc.noaa.gov/oa/climate/research/2002/ann/ann02.html). The 2002 El Niño was also anomalous, with the warm SST anomaly located in the Central rather than Eastern Pacific (Michelle Reinecker, private communication). Such anomalous behavior in both these dry regions and El Niño behavior suggests an unusual forcing of large scale waves in the tropics during 2002. Second, why were waves 2 and 3 heat flux and and amplitudes unusually high, but not in the tropics? From Hovmoller diagrams and coherency plots, a traveling wave 2 pattern is evident over most of the winter with a period of 10-14 days. However, the forcing of this traveling Rossby wave 2 is not apparent. The unusual wave 2 and 3 patterns may be caused by wave-trains forced in the tropics and propagating into the mid-latitudes as was shown shown in Hoskins and Karoly (1981) for the Northern Hemisphere winter.

Planetary wave 1 events are forced in the tropical upper troposphere. These tropically forced waves occur episodically over the course of the SH winter. This wave 1 takes $2-4$ days to propagate across the equatorial region and downward into the lower troposphere near $55^{\circ} \mathrm{S}$. Because the zonal mean flow at the tropopause is conducive to wave propagation, the wave 1 in the lower troposphere is efficiently refracted upward into the stratosphere. Over the course of the winter, these wave events warm the polar lower stratosphere and weaken the jet stream. By mid-September, these series of wave events have pre-conditioned the stratospheric zonal mean wind pattern is pre-conditioned for a major warming. A final large event in late September then results in a major warming of the stratosphere. The warming is strong enough to warm temperatures beyond the formation point for polar stratospheric clouds, thereby stopping ozone loss.

Acknowledgments. We wish to thank Drs. Darryn Waugh, Warrick Norton, Mark Schoeberl, Lorenzo Polvani, and Leslie Gray for helpful discussions. This work was supported under NASA grant xxx from the Atmospheric Chemistry, Modeling, and Analysis Program. 
Sea surface temperatures and the Southern Oscillation Index provided by the NOAA/ National Weather Service National Centers for Environmental Prediction Climate Prediction Center. 


\section{REFERENCES}

Andrews, D. G., J. R. Holton, and C. B. Leovy, 1987: Middle Atmosphere Dynamics. Academic, San Diego, Calif., 489 pp.

Chen, P., and W. A. Robinson, 1992: Propagation of planetary waves between the troposhpere and stratosphere. J. Atmos. Sci., 49, 2533-2545.

Court, A., 1942: Tropopause disappearance during the Antarctic Winter. Bull. Amer. Met. Soc., 23, 220-238.

Dickinson, R. E., 1968: Planetary rossby waves propagating vertically through weak westerly wind wave guide. J. Atmos. Sci., 25, 984-1002.

Gray, L. J., E. F. Drysdale, T. J. Dunkerton, and B. N. Lawrence, 2001a: Model studies of the interannual variability of the northern-hemisphere stratospheric winter circulation: The role of the quasi-biennial oscillation. Quart. J. Roy. Met. Soc., 127, 1413-1432.

-_--, S. J. Phipps, T. J. Dunkerton, M. P. Baldwin, E. F. Drysdale, and M. R. Allen, 2001b: A data study of the influence of the equatorial upper stratosphere on northern-hemisphere stratospheric sudden warmings. Quart. J. Roy. Met. Soc., 127, 1985-2003.

Hartmann, D. L., C. R. Mechoso, and K. Yamazaki, 1984: Observations of wave-mean flow interaction in the southern hemisphere. J. Atmos. Sci., 41, 351-362.

Holton J. R., and H. C. Tan, 1982: The quasi-biennial oscillation in the northern hemisphere lower stratosphere. J. Meteorol. Soc. Japan, 60, 140-148.

Kanamitsu, M., R. E. Kistler, and R. W. Reynolds, 1997: NCEP/NCAR reanalysis and the use of satellite data. Adv. Space Res., 19, 481-489.

Marshall, G. J., 2002: Trends in Antarctic geopotential height and temperature: A comparison between radiosonde and NCEP-NCAR reanalysis data. J. Climate, 15, 659-674.

Mo, K. C., X. L. Wang, R. Kistler, M. Kanamitsu, and E. Kalnay, 1995: Impact of satellite data on the CDAS reanalysis system. Mon. Wea. Rev., 123, 124-139.

Newman, P. A., and E. R. Nash, 2000: Quantifying the wave driving of the stratosphere. 
J. Geophys. Res., 105, 12,485-12,497.

E. R. Nash, and J. E. Rosenfield, 2001: What controls temperature of the Arctic stratosphere during spring? J. Geophys. Res., 106, 19,999-20,010.

Randel, W. J., 1992: Global atmospheric circulation statistics, 1000-01 mb. NCAR Tech. Note, NCAR/TN-366+STR, $256 \mathrm{pp}$.

-...-, F. Wu, and R. Stolarski, 2002: Changes in Column Ozone Correlated with the Stratospheric EP Flux. J. Met. Soc. Japan, 80, 849-862.

Santer, B. D., J. J. Hnilo, T. M. L. Wigley, J. S. Boyle, C. Doutriaux, M. Fiorino, D. E. Parker, and K. E. Taylor, 1999: Uncertainties in observationally based estimates of temperature change in the free atmosphere. J. Geophys. Res., 104, 6305-6333.

Scinocca, J. F., and P. H. Haynes, 1998: Dynamical forcing of stratospheric planetary waves by tropospheric baroclinic eddies. J. Atmos. Sci., 55, 2361-2392.

Scott, R. K., and P. H. Haynes, 1998: Internal interannual variability of the extratropical stratospheric circulation: The low-latitude flywheel. Q. J. Roy. Meteor. Soc., 124, 2149-2173.

Shiotani, M., and J. C. Gille, 1987: Dynamic factors affecting ozone mixing ratios in the antarctic lower stratosphere. J. Geophys. Res., 92, 9811-9824.

Stolarski, R. S., and Coauthors, 2003: The 2002 Antarctic Ozone hole.

Taguchi, M., and S. Yoden, 2002: Internal interannual variability of the tropospherestratosphere coupled system in a simple global circulation model. Part I: Parameter sweep experiment. J. Atmos. Sci., 59, 3021-3036.

Thompson, D. W. J., S. Solomon, and D. J. Hofmann, 2003: An unprecedented southern hemisphere spring. Sci. Brev.

World Meteorological Organization, 1995: Scientific Assessment of Ozone Depletion: 1994. Rep. 37, Global Ozone Res. and Monitor. Proj., Geneva, Switzerland. 
Figure 1. Daily minimum temperature in the region $50^{\circ}-90^{\circ} \mathrm{S}$ at (A) $10 \mathrm{hPa}$ and (B) $50 \mathrm{hPa}$. (C) Daily zonal mean temperature at $65^{\circ} \mathrm{S}$ and $50 \mathrm{hPa}$. The black line in each panel shows the 2002 temperature. The white line displays the 23-year average (1979-2001) and is smoothed with a 15-day boxcar. The gray shading indicates the range of values observed between 1979 and 2001. The vertical gray lines passing through all panels indicate the days of maximum eddy heat flux at $100 \mathrm{hPa}$.

Figure 2. (A) Daily temperatures for 1 April to 31 October 2002 , averaged for $55^{\circ}-75^{\circ} \mathrm{S}$ at 50 hPa. Contour intervals are $2 \mathrm{~K}$. (B) Daily eddy heat flux at $100 \mathrm{hPa}$, averaged for $40^{\circ}-70^{\circ} \mathrm{S}$. Units are in $\mathrm{K} \mathrm{m} \mathrm{s}^{-1}$ (note reversed scale) and values have been smoothed with a 1-2-1 filter. (C) Daily zonal-mean zonal winds, averaged for $20^{\circ}-90^{\circ} \mathrm{S}$. Contour intervals are $2 \mathrm{~m} \mathrm{~s}^{-1}$ and negative contours are dashed. The vertical lines passing through all panels indicate the days of maximum eddy heat flux at $100 \mathrm{hPa}$.

Figure 3. (a) Temperature at $50 \mathrm{hPa}$ and averaged for $60^{\circ}-90^{\circ} \mathrm{S}$ plotted against eddy heat flux at $100 \mathrm{hPa}$ and averaged for $45^{\circ}-75^{\circ} \mathrm{S}$. (A) Temperature averaged for $16-30$ September and eddy heat flux averaged for 2 August to 23 September. (B) Temperature averaged for 1-15 September and eddy heat flux averaged for 18 July to 8 September. The solid line is the leastsquares regression fit and the correlations are indicated. Each year is indicated by the last two digits.

Figure 4. (A) July zonal-mean temperature departures from the long-term mean (1979-2001). Contour intervals are $1 \mathrm{~K}$. The white contours are the long-term mean field, with intervals of 5 K. (B) July zonal-mean zonal wind departures from the long-term mean (1979-2001). Contour intervals are $2 \mathrm{~m} \mathrm{~s}^{-1}$. The white contours show the long-term mean field, with intervals of 10 $\mathrm{m} \mathrm{s}^{-1}$. Negative contours are dashed in both panels. 
Figure 5. (A) Daily Eliassen-Palm (EP) flux vectors for waves 1-3 averaged from $30^{\circ}-70^{\circ} \mathrm{S}$. The vectors have been multiplied by the log of pressure to emphasize the lower altitude values. Vectors pointing up indicate vertical propagation while vectors pointing to the right indicate equatorward propagation. Daily EP flux vectors at $300 \mathrm{hPa}$ (B) $10 \mathrm{hPa}$ (C). In these lower 2 panels, vectors pointing upward indicate equatorward propagation while vectors pointing to the left indicate upward propagation. In all 3 panels, EP flux vectors less than 20

Figure 6. (A) Pressure-time plot of daily geopotential height RMS waves 1-3 amplitude averaged over latitudes $45^{\circ}-75^{\circ} \mathrm{S}$. The RMS wave amplitude is normalized by the density. The horizontal white line shows the $300 \mathrm{hPa}$ level. Contour intervals are $25 \mathrm{~m}$. (B) Latitude-time plot of daily geopotential height RMS waves 1-3 amplitude at $300 \mathrm{hPa}$. The white horizontal lines indicate the $45^{\circ}$ and $75^{\circ} \mathrm{S}$ latitude lines used in panel $\mathrm{A}$, while the horizontal gray line is the $60^{\circ} \mathrm{S}$ latitude line. Contour intervals are $100 \mathrm{~m}$. (C) Longitude-time plot of daily geopotential height waves $1-3$ deviations from the zonal mean at $60^{\circ} \mathrm{S}$ and $300 \mathrm{hPa}$. Contour intervals are $300 \mathrm{~m}$. The vertical lines passing through all panels indicate the days of maximum eddy heat flux at $100 \mathrm{hPa}$.

Figure 7. Contour plot of the $100 \mathrm{hPa}, 60^{\circ} \mathrm{S}$ wave 1 coherency plotted as a function of pressure and time lag. Coherency is similar to a correlation coefficient, and represents linearly related temporal variability between points. The white lines are drawn onto the axis of the maximum of the coherency to illustrate the vertical propagation of wave 1. Contour intervals are 0.1 .

Figure 8. Contour plots of the $150 \mathrm{hPa}, 30^{\circ} \mathrm{S}$ wave 1 coherency plotted as a function of altitude and latitude for phase lags of (A) -4 days, (B) 0 days, (C) +2 days, and (D) +4 days. The white lines are drawn onto the axis of the maximum of the coherency to illustrate the propagation of wave 1 . Contour intervals are 0.1 .

Figure 9. Amplitude of wave 1 stream function for 1979-2002 averaged over the period of 1 May to 15 September for each year. (A) Stream function at $700 \mathrm{hPa}$ and averaged for $50^{\circ}-70^{\circ} \mathrm{S}$. (B) Stream function at $300 \mathrm{hPa}$ and averaged for $10^{\circ}-20^{\circ} \mathrm{S}$. The stream function is derived from the non-divergent part of the meridional and zonal winds on pressure surfaces. 
Figure 10. Eddy heat flux vs. the meridional gradient of the quasi-geostrophic potential vorticity $\left(Q_{y}\right)$ averaged over the period of 1 May to 15 September for each year. The heat flux is averaged for $45^{\circ}-75^{\circ} \mathrm{S}$ at $100 \mathrm{hPa}$, while $Q_{y}$ is averaged for $40^{\circ}-60^{\circ} \mathrm{S}$ and $100-400 \mathrm{hPa}$. The index of refraction is proportional to $Q_{y}$. The correlation is 0.55 . Each year is indicated by the last two digits. 
Table 1. Significant 2002 wave events as indicated by the $100-\mathrm{hPa}$ eddy heat flux departures from the 1979-2001 daily means averaged over $40^{\circ}-70^{\circ} \mathrm{S}$.

\begin{tabular}{lccccccc}
\hline Date & NDAYS & TOTAL & W1 & W2 & W3 & WN & $\Delta T$ \\
\hline & & & & & & & \\
15 May & 5 & 4.89 & 4.55 & 0.63 & -0.29 & 1 & 1.7 \\
9 June & 4 & 8.88 & -0.13 & $\mathbf{8 . 0 2}$ & 0.99 & 2 & 1.4 \\
24 June & 4 & 6.00 & 0.47 & $\mathbf{3 . 2 9}$ & 2.24 & 2,3 & 1.6 \\
8 July & 7 & 9.54 & $\mathbf{6 . 3 1}$ & 2.99 & 0.24 & 1 & 1.7 \\
19 July & 6 & 2.13 & -2.28 & 2.02 & $\mathbf{2 . 3 9}$ & 2,3 & 2.1 \\
31 July & 11 & 3.75 & 0.06 & $\mathbf{4 . 8 6}$ & -1.17 & 2 & 3.1 \\
18 August & 11 & 8.77 & $\mathbf{8 . 3 1}$ & -0.56 & 1.01 & 1 & 9.2 \\
4 September & 6 & 4.20 & -3.48 & $\mathbf{8 . 3 7}$ & -0.69 & 2 & 4.4 \\
11 September & 3 & -4.40 & -5.59 & -1.46 & $\mathbf{2 . 6 5}$ & 2,3 & 1.6 \\
22 September & 5 & 35.82 & 13.00 & $\mathbf{1 4 . 6 2}$ & 8.20 & $1,2,3$ & 18.4 \\
25 October & 8 & 5.31 & $\mathbf{4 . 1 2}$ & -0.12 & 1.31 & 1 & 1.2 \\
& & & & & & & \\
\hline
\end{tabular}

Date $=$ Date when the peak in waves 1-3 eddy heat flux is maximum.

NDAYS $=$ The number of days in an event is determined by the when the sign of the first derivative changes on either side of the peak.

TOTAL, W1, W2, W3 = Total, wave 1, wave 2, and wave 3 amplitude of eddy heat flux $\left(\mathrm{K} \mathrm{m} \mathrm{s}^{-1}\right)$.

WN = Dominant wave number(s) for the event.

$\Delta \mathrm{T}=$ Difference in 50-hPa temperature departures from the 1979-2001 daily means, averaged over $60^{\circ}-90^{\circ} \mathrm{S}$, between the maximum value after the event and the value at the start of the event, defined by the criteria used for NDAYS (K). 
Fig. 1

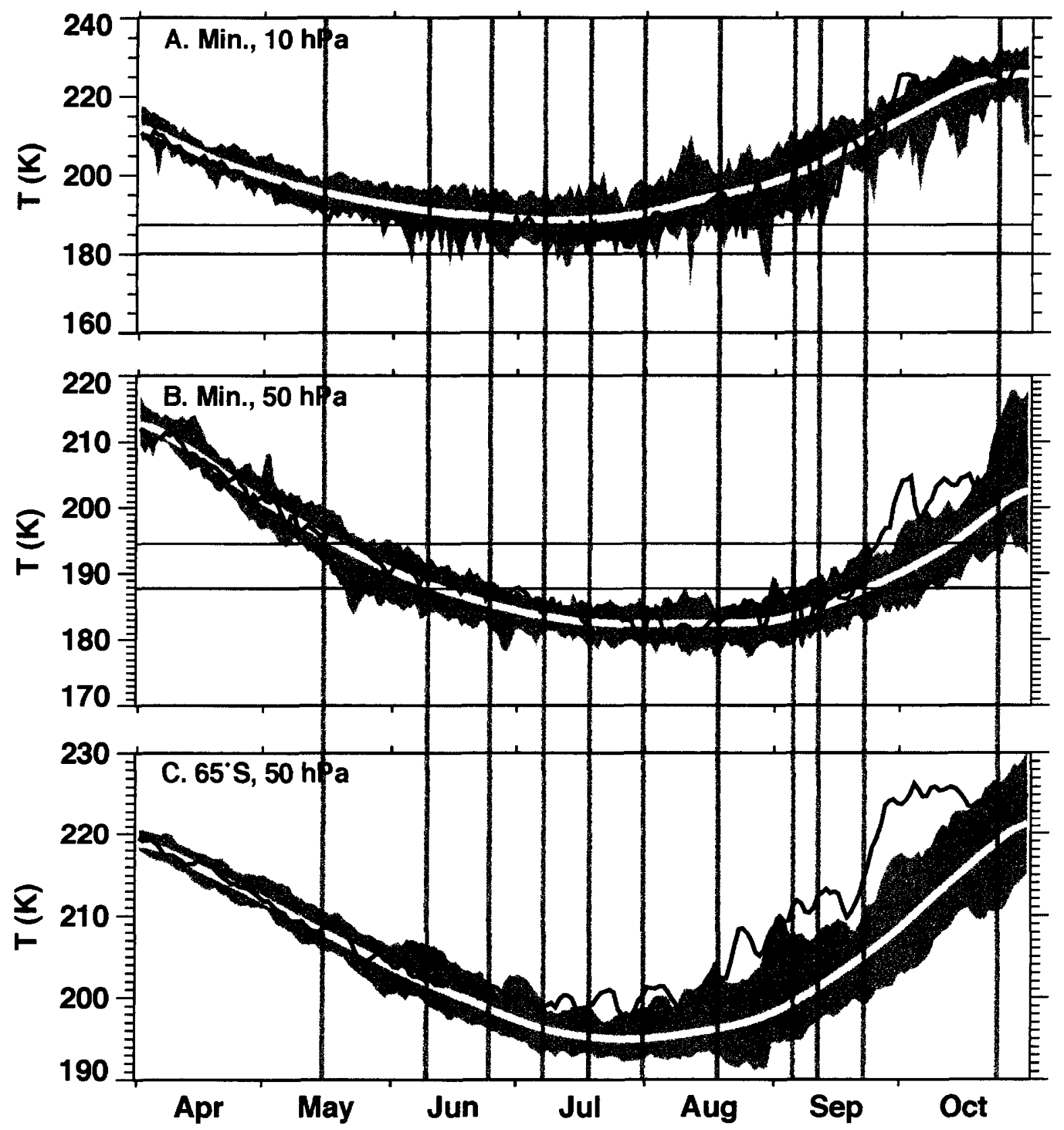


Fig. 2

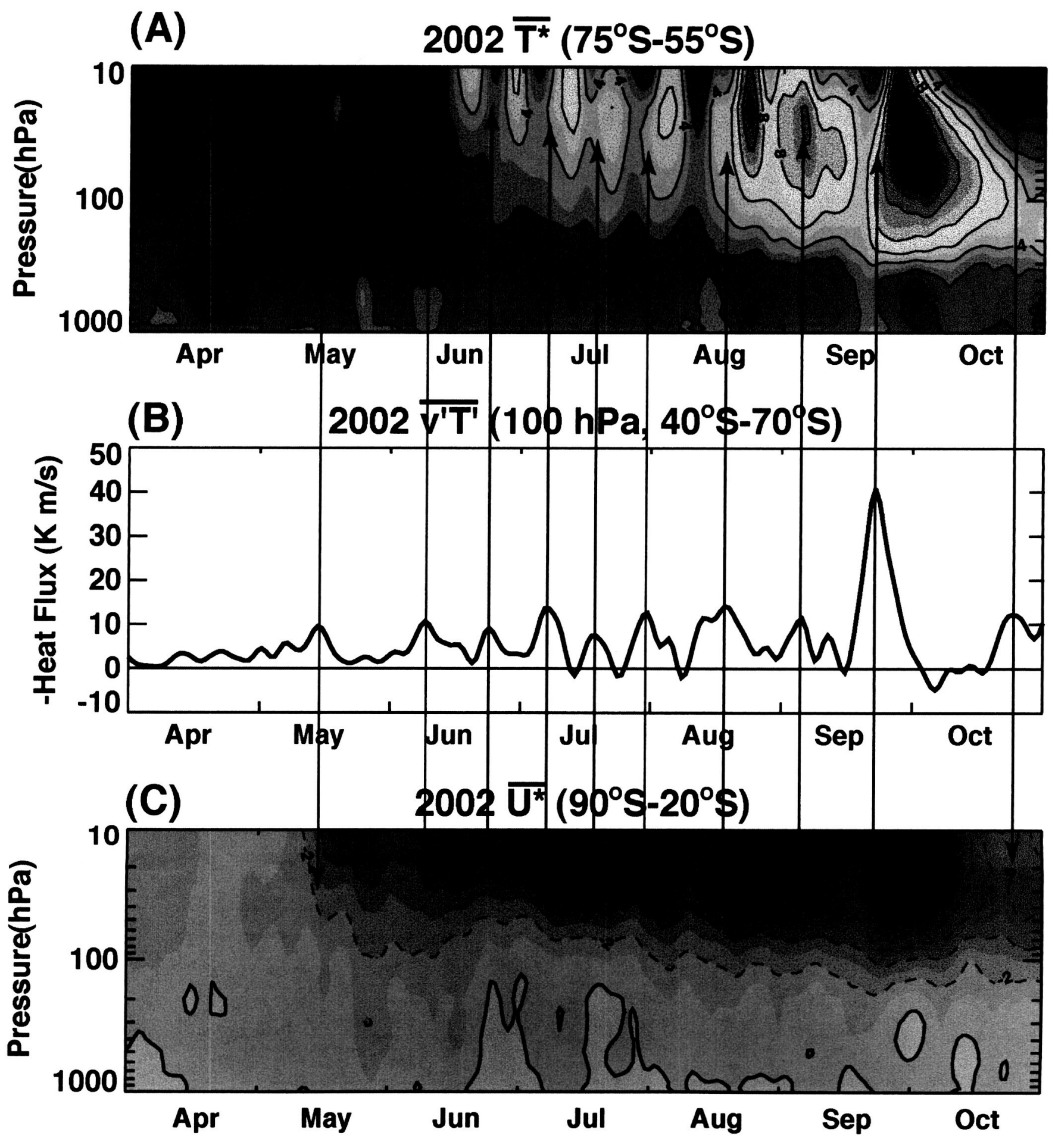


Fig. 3
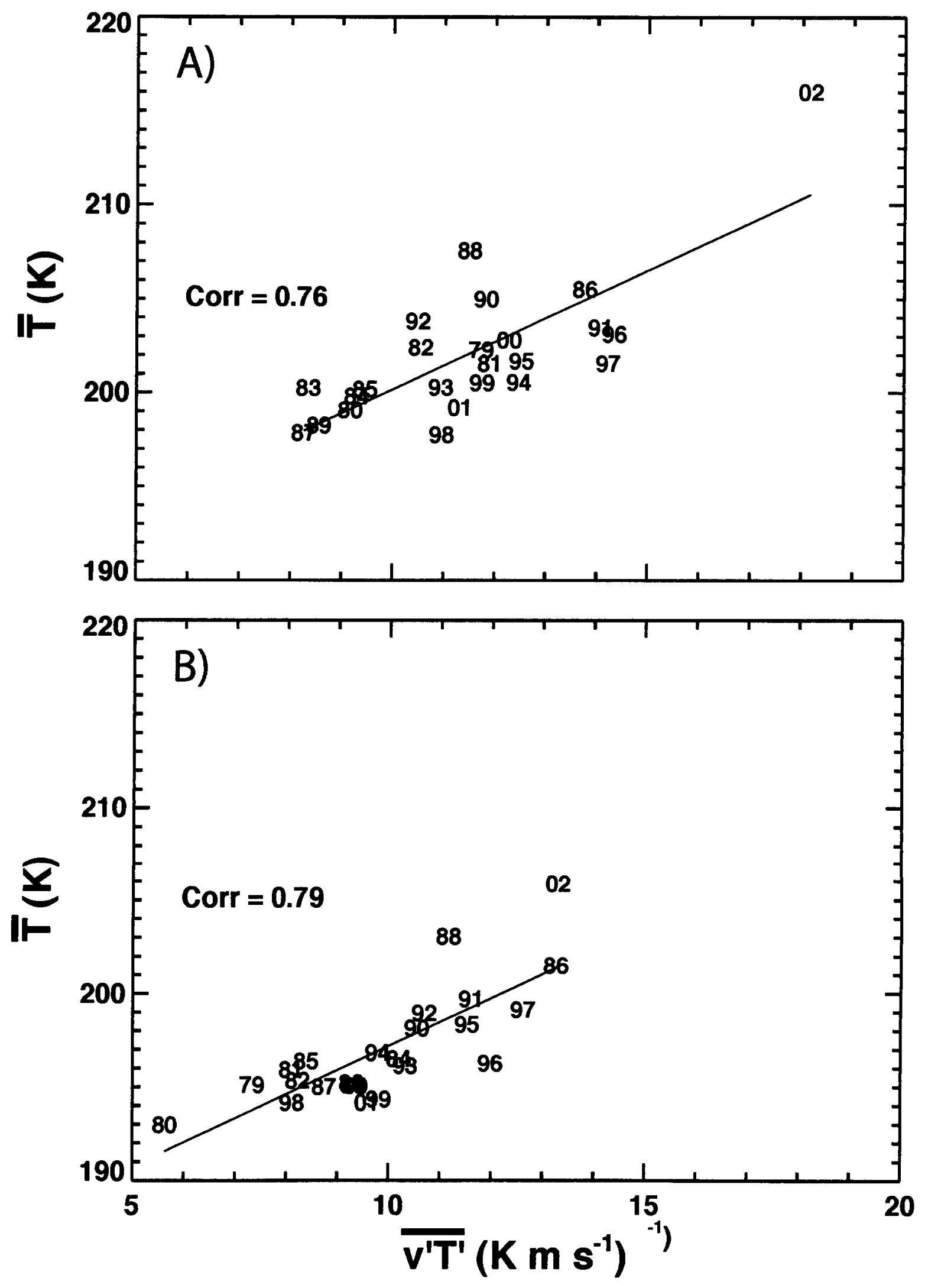
Fig. 4
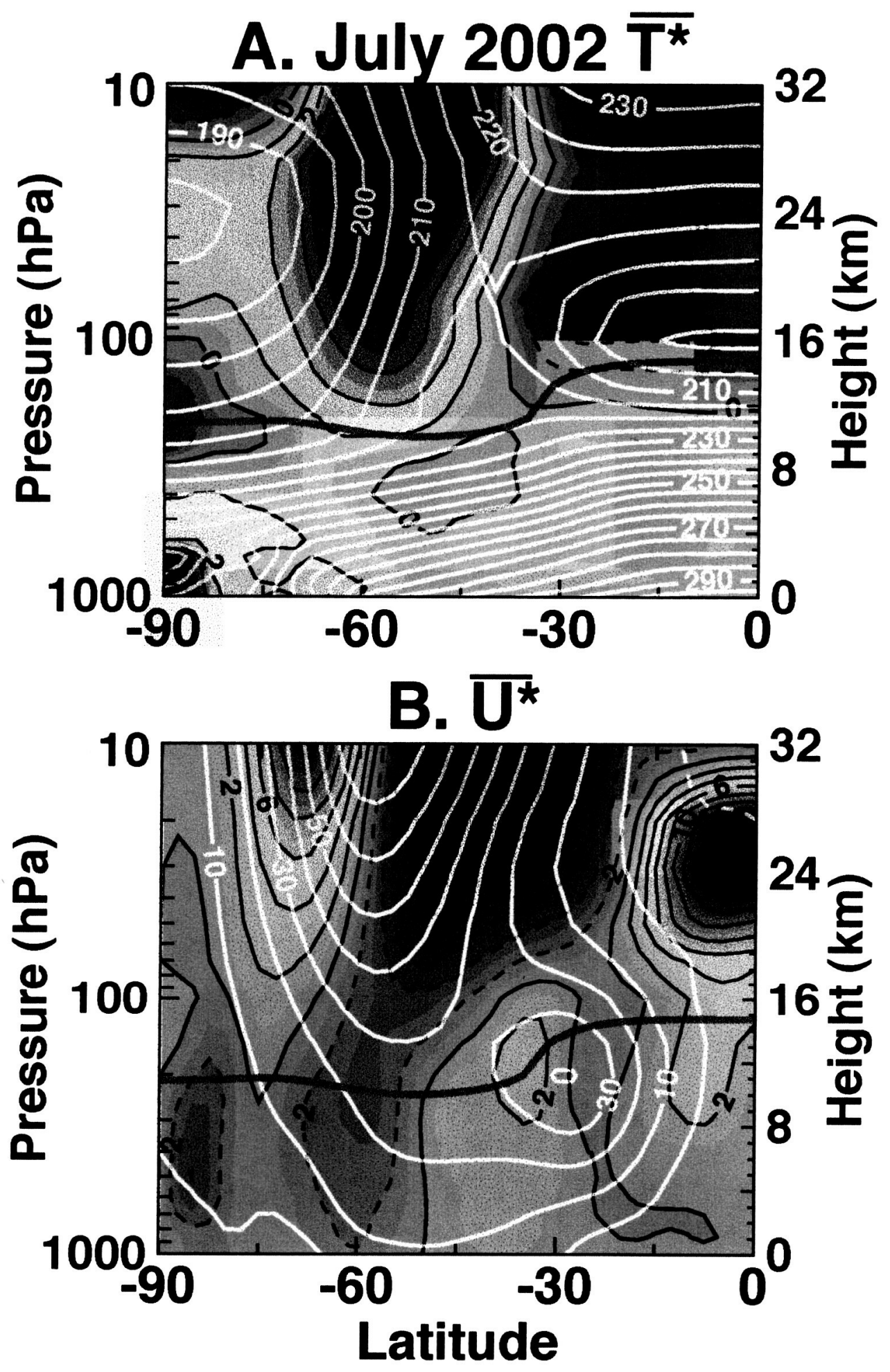
Fig. 5
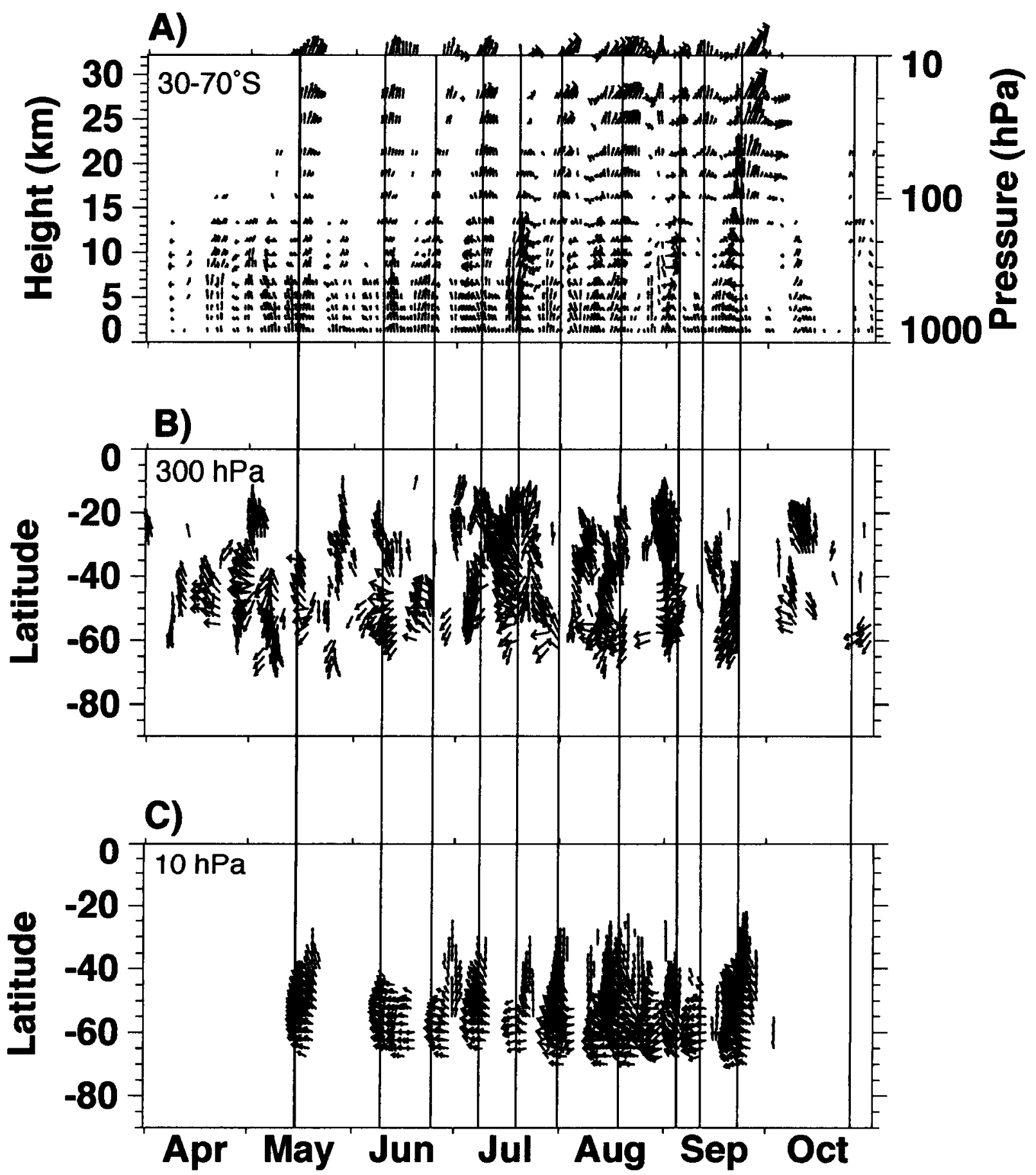

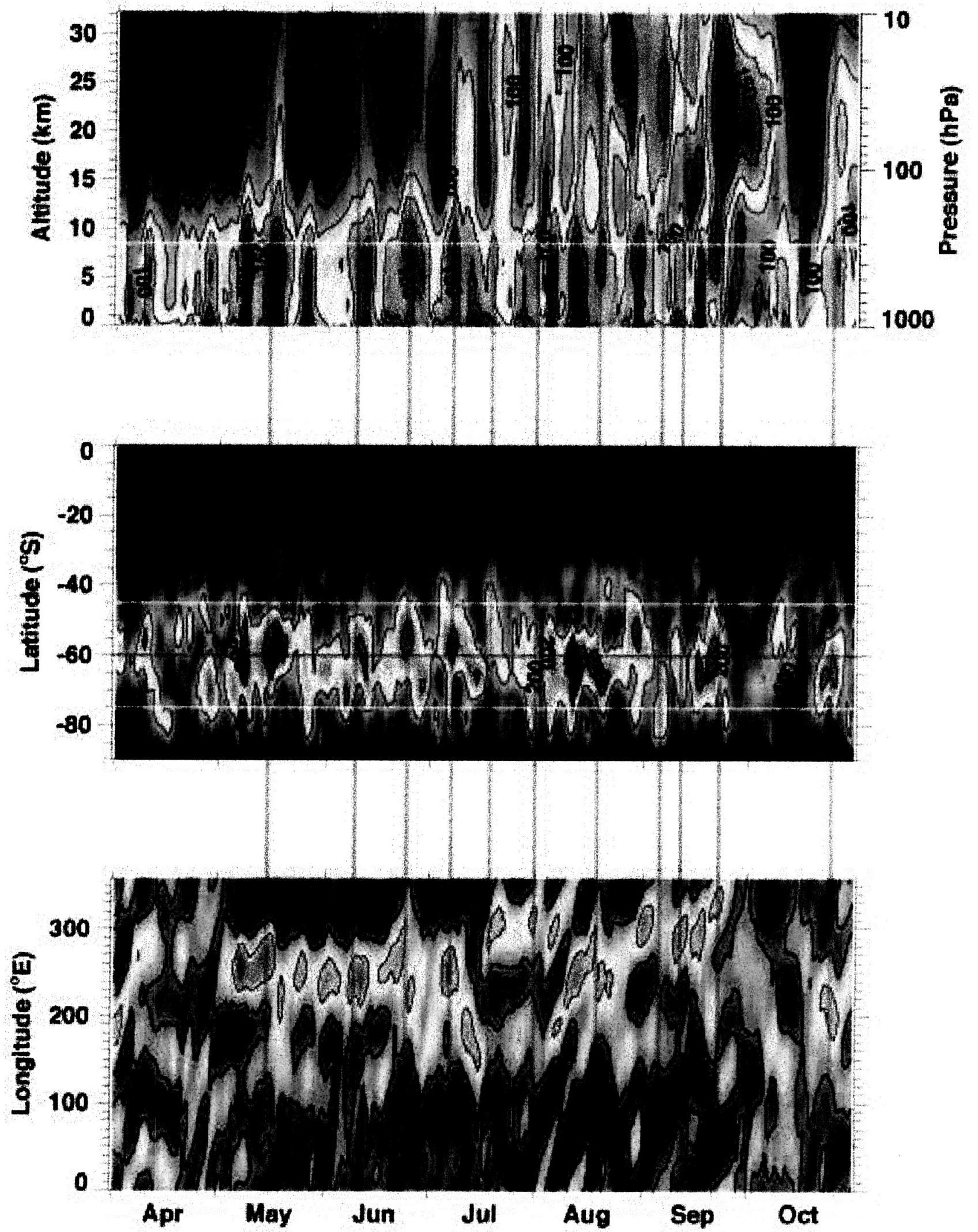
Figure 7

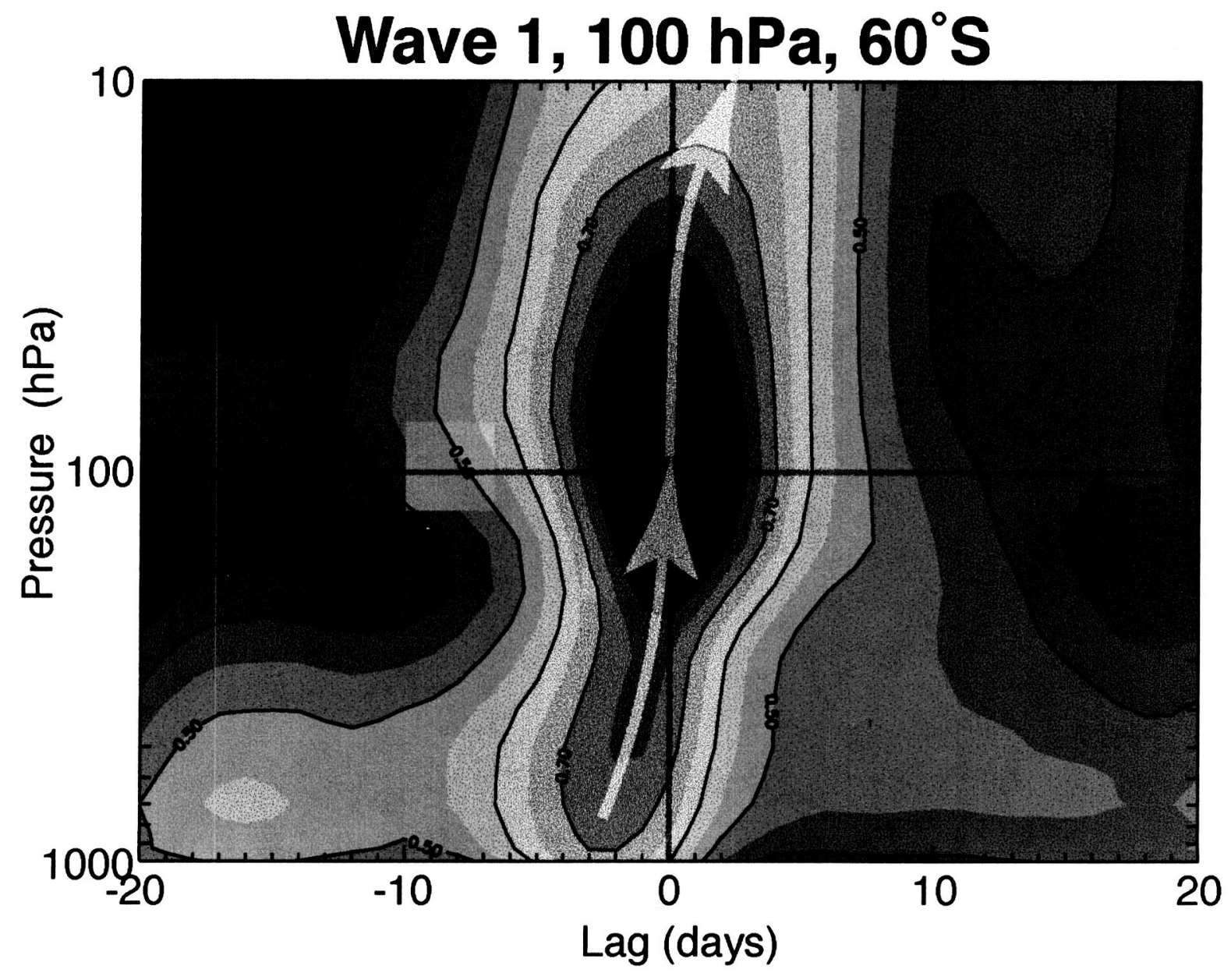


Figure 8
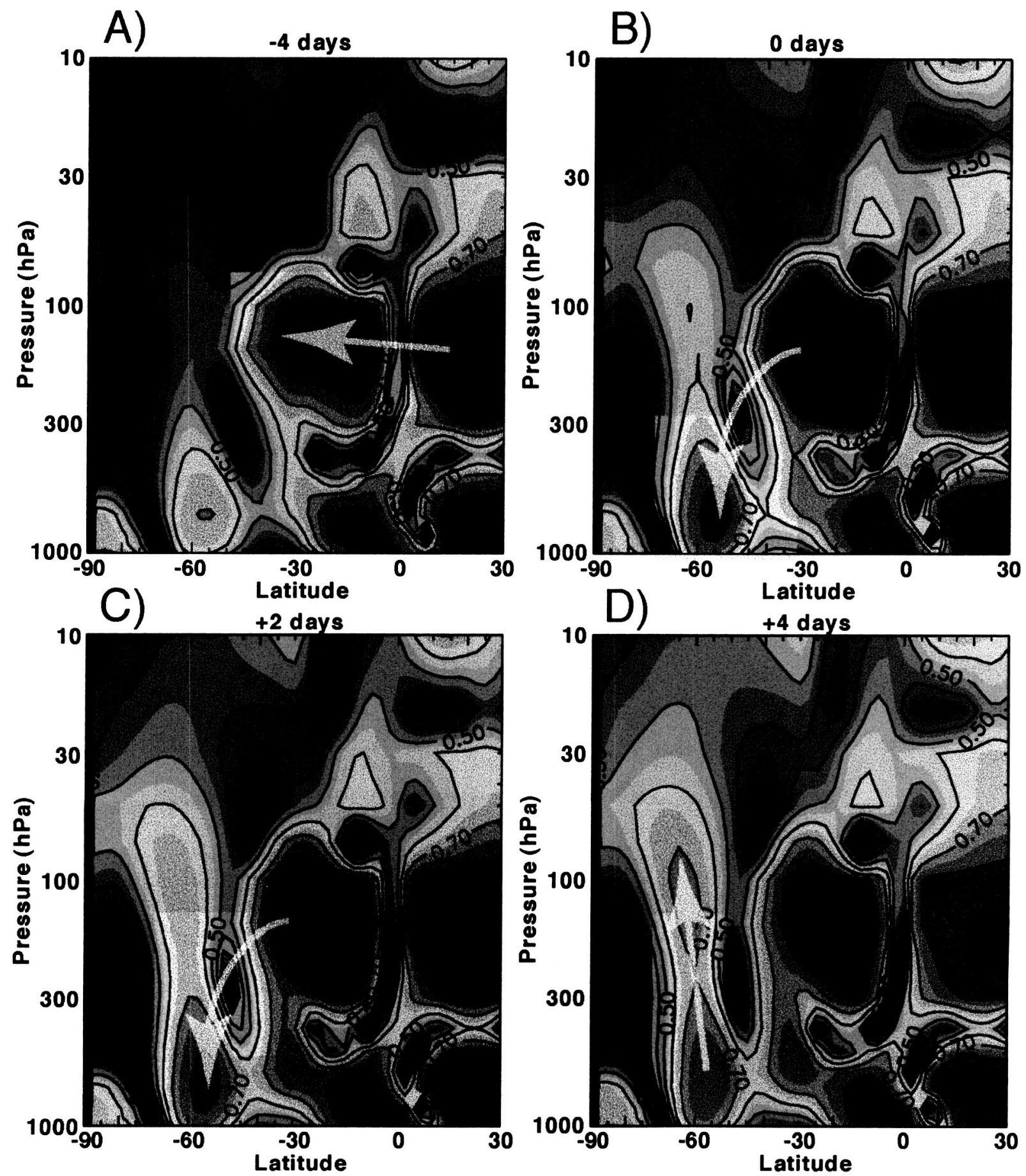
Figure 9
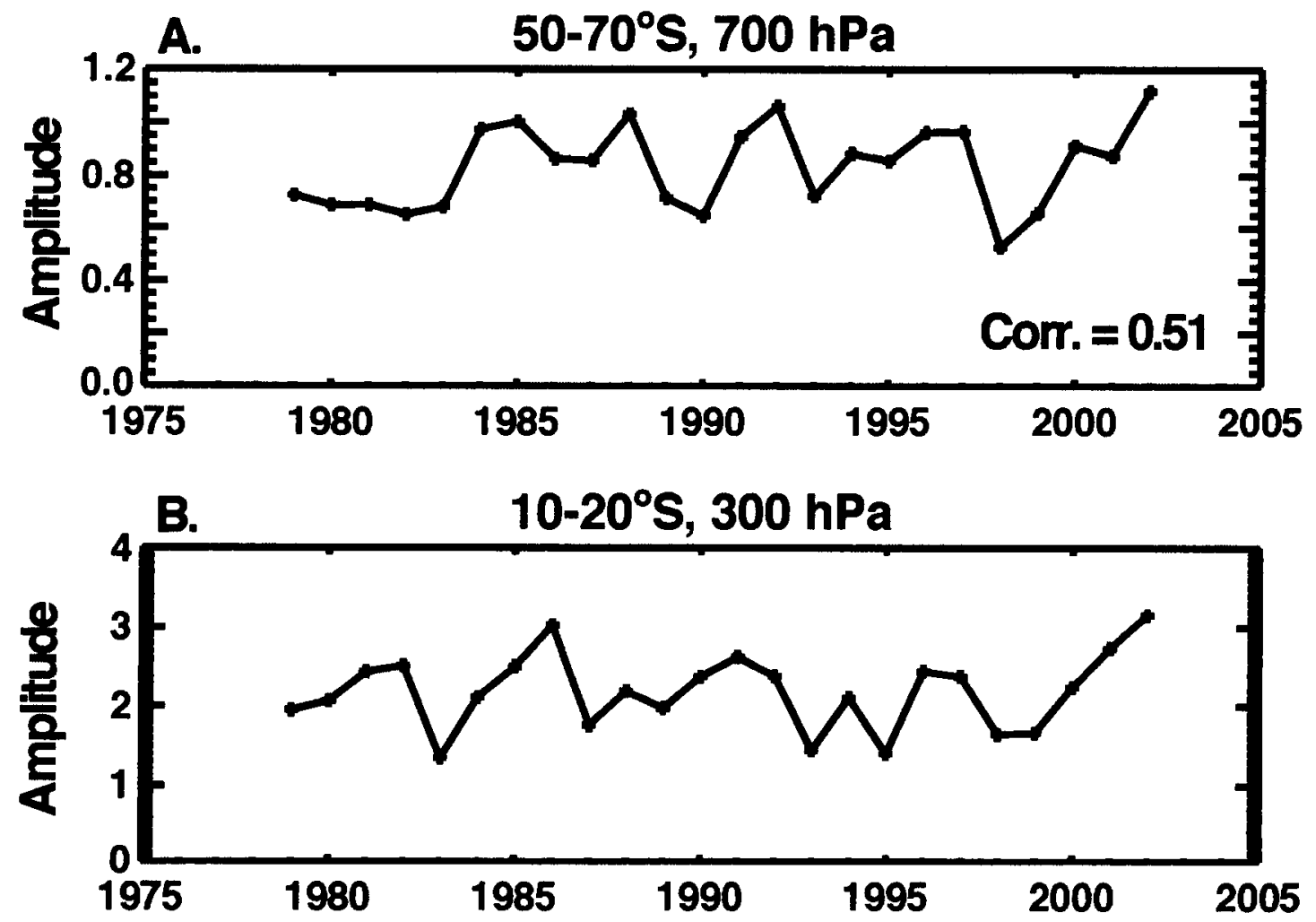
Figure 10

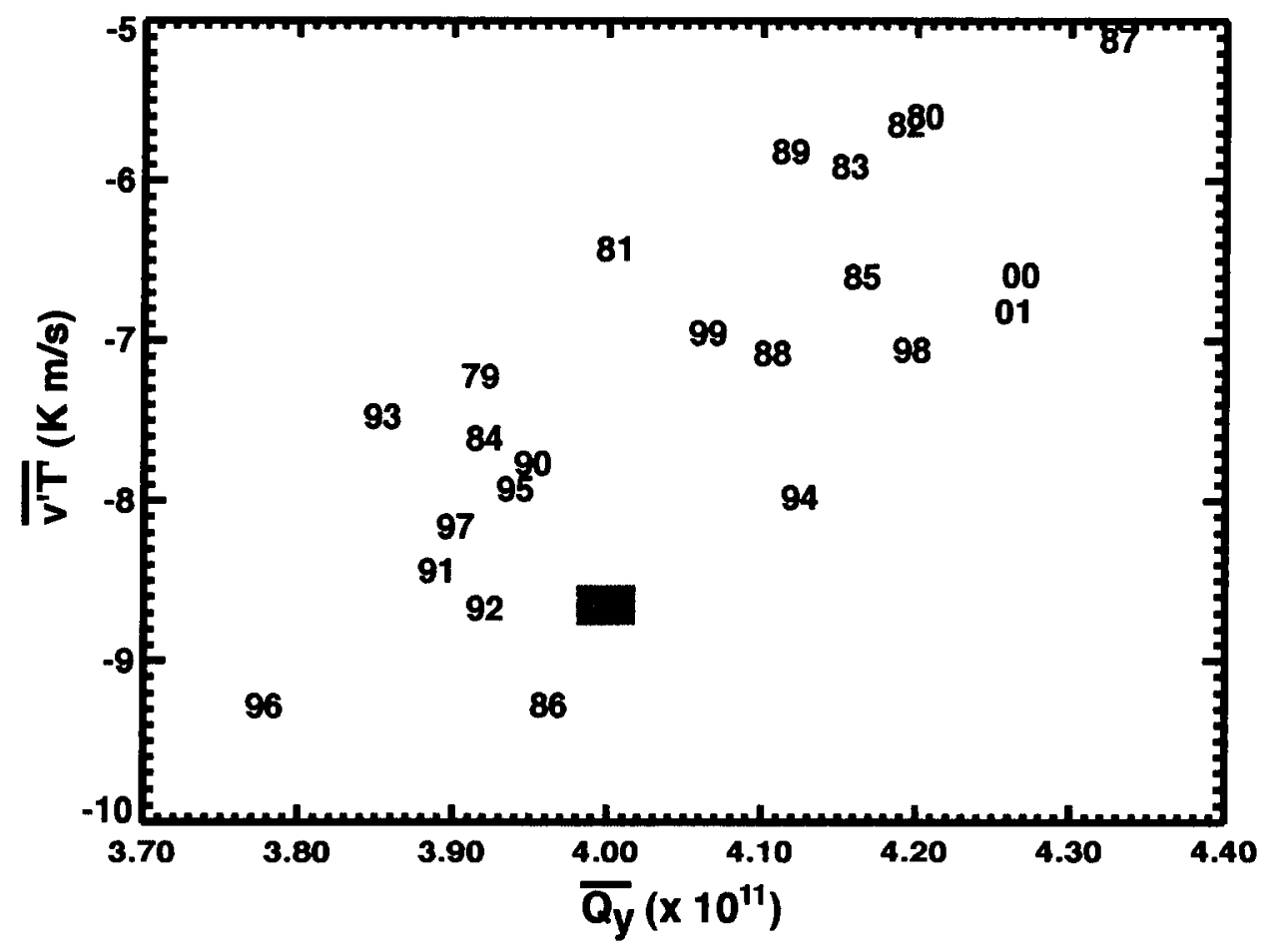

